TITLE:

\title{
Conditions for predicting quasistationary states by rearrangement formula
}

$\operatorname{AUTHOR}(S)$ :

Yamaguchi, Yoshiyuki Y.; Ogawa, Shun

CITATION:

Yamaguchi, Yoshiyuki Y....[et al]. Conditions for predicting

quasistationary states by rearrangement formula. Physical Review E 2015, 92(4): 042131.

ISSUE DATE:

2015-10-14

URL:

http://hdl.handle.net/2433/200621

RIGHT:

(C)2015 American Physical Society. 
PHYSICAL REVIEW E 92, 042131 (2015)

\title{
Conditions for predicting quasistationary states by rearrangement formula
}

\author{
Yoshiyuki Y. Yamaguchi* \\ Department of Applied Mathematics and Physics, Graduate School of Informatics, Kyoto University, 606-8501 Kyoto, Japan \\ Shun Ogawa ${ }^{\dagger}$ \\ Aix-Marseille Université, Université de Toulon, CNRS, Centre de Physique Théorique UMR 7332, 13288 Marseille Cedex 9, France \\ (Received 26 November 2014; revised manuscript received 9 February 2015; published 14 October 2015)
}

\begin{abstract}
Predicting the long-lasting quasistationary state for a given initial state is one of central issues in Hamiltonian systems having long-range interaction. A recently proposed method is based on the Vlasov description and uniformly redistributes the initial distribution along contours of the asymptotic effective Hamiltonian, which is defined by the obtained quasistationary state and is determined self-consistently. The method, to which we refer as the rearrangement formula, was suggested to give precise prediction under limited situations. Restricting initial states consisting of a spatially homogeneous part and small perturbation, we numerically reveal two conditions that the rearrangement formula prefers: One is a no Landau damping condition for the unperturbed homogeneous part, and the other comes from the Casimir invariants. Mechanisms of these conditions are discussed. Clarifying these conditions, we validate to use the rearrangement formula as the response theory for an external field, and we shed light on improving the theory as a nonequilibrium statistical mechanics.
\end{abstract}

DOI: 10.1103/PhysRevE.92.042131

PACS number(s): 05.20.Dd, 46.40.Ff

\section{INTRODUCTION}

Long-range interaction violates some assumptions introduced in the equilibrium statistical mechanics and thermodynamics, for instance additivity [1,2]. One remarkable phenomenon in such a system is the existence of long-lasting nonequilibrium quasistationary states (QSSs) in the relaxation process, and the lifetime of QSSs diverges in the limit of large population [3-6]. In the mean-field limit, dynamics of the system is described by the Vlasov equation (or collisionless Boltzmann equation) [7-9], and QSSs are regarded as stable stationary solutions to the Vlasov equation. QSSs are said to be found in various scales in the nature, from the laboratory scale as the plasma crystals [10-12] to extremely large scale as the elliptic or spherical galaxies [3]. A central issue of long-range interacting systems is to predict the QSS from a given nonstationary initial state.

One theoretical approach is proposed by Lynden-Bell [13], which is originally proposed for the self-gravitating systems and is easy to use for the so-called waterbag initial states. Several tests are performed for the theory in the self-gravitating systems with one dimension [14,15], two dimensions [16], and three dimensions [17], and the Hamiltonian mean-field (HMF) model (or the globally coupled $X Y$ model) [18]. In the selfgravitating systems, thanks to homogeneity of potential, initial states are classified by the virial ratio, and the Lynden-Bell's theory gives good prediction of QSSs if initial states satisfy the virial condition. The concept of virialization is extended for nonhomogeneous potential of the HMF model [18] to avoid parametric resonance making halo $[19,20]$. The generalized virial condition helps to prepare initial states for which QSSs are described by the Lynden-Bell's theory. See also Ref. [21].

\footnotetext{
*yyama@amp.i.kyoto-u.ac.jp

${ }^{\dagger}$ On leave from Department of Applied Mathematics and Physics, Kyoto University; shun.ogawa@cpt.univ-mrs.fr
}

Another approach is the rearrangement formula, or the integrable (uncoupled) model. In this article we consider asymptotic states of the Vlasov dynamics which are QSSs in which we are interested. The idea to get the asymptotic state is to redistribute the initial distribution along contours of the asymptotic effective Hamiltonian, which is determined by the asymptotic state, and to solve the self-consistent equation for the asymptotic state. The rearrangement formula is introduced without theoretical justification, but is successfully examined in the HMF model for single-level [22,23] and multilevel [24] waterbag initial states numerically. Further, the theory also gives good prediction for three-dimensional self-gravitating systems for the waterbag initial states, and for the parabolic initial states [25].

The two different theories of the rearrangement formula and of Lynden-Bell prefer the generalized virial states, but the former is said to provide more accurate predictions than the latter [24,25]. We then focus on the rearrangement formula rather than the Lynden-Bell's theory. Another reasoning to focus on the rearrangement formula is that the formula is useful even for nonwaterbag initial states. Indeed, for perturbed states from stable stationary states, disordered thermal equilibria for instance, the rearrangement formula is theoretically justified [26] by the use of the asymptotic-transient field decomposition and the transient (T-)linearized Vlasov equation [27-29]. We note that the naming is "linearization," but the equation includes a nonlinear term as shown later. A similar formula is also derived via the variational principle in the context of plasma waves [30,31]. However, as Lynden-Bell's theory, the rearrangement formula is not always precise and a previous work [26] suggests that the stable stationary state with zero Landau damping [32] rate is preferred as the unperturbed states. We refer to this condition as the no Landau damping condition.

It is still unclear if the no Landau damping condition is more relevant than the virial condition, and if the former is solely essential, since numerical tests have been performed for a limited situation. Moreover, the no Landau damping condition 
is for the unperturbed states, and hence one may expect a condition for the whole initial state including perturbation. The main purposes of the present article are to confirm the relevance of the no Landau damping condition and to reveal one more condition relating to the Casimir invariance by performing systematic numerical simulations of the Vlasov equation.

It is important to clarify validating conditions of the rearrangement formula from the following two contexts. One is as the response theory for external field. The rearrangement formula gives nonclassical critical exponents, and the theoretical predictions are in good agreement with numerical simulations $[26,33,34]$. We can further justify the nonclassical critical exponents by showing that the validating conditions are satisfied for computing the response. The other is related to improvement of the theory. After confirming the validating conditions, it might be possible to improve the theory by including the Landau damping into the rearrangement formula, for instance.

This article is organized as follows. We first introduce the HMF model and the associated Vlasov equation in Sec. II. In Sec. III, we briefly review the rearrangement formula, and give theoretical predictions for the HMF model. Section IV is for examinations of the two conditions: In Sec. IV A we explain why one may expect the conditions. The no Landau damping condition for the reference state is carefully confirmed in Sec. IV B, and a new condition of the Casimir invariance is reported in Sec. IV C. Based on the numerical findings, we discuss validity for using the rearrangement formula as the response theory to the external force, in particular to compute the critical exponents, in Sec. V. The final Sec. VI is devoted to a summary and discussions.

\section{HAMILTONIAN MEAN-FIELD MODEL AND VLASOV EQUATION}

The HMF model $[35,36]$ is a model of a ferromagnetic body, and is expressed by the Hamiltonian

$$
\begin{aligned}
H_{N}= & \sum_{i=1}^{N} \frac{p_{i}^{2}}{2}+\frac{1}{2 N} \sum_{i, j=1}^{N}\left[1-\cos \left(q_{i}-q_{j}\right)\right] \\
& -\sum_{i=1}^{N}\left[h_{x}(t) \cos q_{i}+h_{y}(t) \sin q_{i}\right],
\end{aligned}
$$

where the last two terms express the interaction energy between $X Y$ spins (rotators) and the external magnetic field $\left(h_{x}(t), h_{y}(t)\right)$. Response to the external field will be discussed in Sec. V, and until then, the external field is set as zero. The system is also looked on as a dynamical system with many particles moving on the unit circle with attractive all-to-all interactions, and the position and the conjugate momentum of the $i$ th particle are denoted by $q_{i}$ and $p_{i}$ respectively defined in $q_{i} \in(-\pi, \pi]$ and $p_{i} \in \mathbb{R}$. The HMF model is a paradigmatic toy model, and the simple interaction provides advantages in theory and in numerics.

When one takes the limit of $N \rightarrow \infty$, temporal evolution of the HMF model can be well described in terms of the single particle distribution $f(q, p, t)$ governed by the Vlasov equation
[7-9]

$$
\partial_{t} f+\{\mathcal{H}[f], f\}=0, \quad f(q, p, 0)=f_{\mathrm{I}}(q, p),
$$

where the Poisson bracket $\{a, b\}$ is defined as

$$
\{a, b\}=\frac{\partial a}{\partial p} \frac{\partial b}{\partial q}-\frac{\partial a}{\partial q} \frac{\partial b}{\partial p}
$$

for two functions on the $\mu$ space $(-\pi, \pi] \times \mathbb{R}$. The effective Hamiltonian $\mathcal{H}[f]$ is given by

$$
\mathcal{H}[f]=p^{2} / 2+\mathcal{V}[f](q, t)
$$

with

$$
\mathcal{V}[f]=-\left(\mathcal{M}_{x}[f]+h_{x}\right) \cos q-\left(\mathcal{M}_{y}[f]+h_{y}\right) \sin q .
$$

In this article, we look into the dynamics through the magnetization (or the order parameter) vector $\left(\mathcal{M}_{x}[f], \mathcal{M}_{y}[f]\right)$ defined by

$$
\mathcal{M}_{x}[f]+i \mathcal{M}_{y}[f]=\iint e^{i q} f(q, p, t) d q d p .
$$

The magnetization vector has the modulus less than or equal to 1 , and measures how particles concentrate at a certain direction on the circle. If particles are uniformly distributed, then $\mathcal{M}_{x}[f]=\mathcal{M}_{y}[f]=0$. If particles are squeezed at a point on the unit circle, for instance $q=0$, then $\mathcal{M}_{x}[f]=1$ and $\mathcal{M}_{y}[f]=0$.

\section{REARRANGEMENT FORMULA}

\section{A. General derivation}

We shortly review the rearrangement formula in the absence of the external field. See [26] for theoretical justification of the formula.

We start from the initial state $f_{\mathrm{I}}$ close to a spatially homogeneous stable stationary state $f_{\mathrm{S}}$, and decompose it into the two parts as

$$
f_{\mathrm{I}}(q, p)=f_{\mathrm{S}}(p)+\epsilon g_{\mathrm{I}}(q, p), \quad|\epsilon| \ll 1 .
$$

We note that we can construct the rearrangement formula even if the unperturbed part is spatially inhomogeneous, but we restrict ourselves to the homogeneous case for simplicity. Our interest is to predict the asymptotic state of the Vlasov dynamics denoted by $f_{\mathrm{A}}$, which is assumed to be stationary. It should be noted that the perturbed state (7) possibly does not go to a stationary state, but to an oscillatory state by forming small traveling clusters under some conditions [37]. We do not look into such states in the present article.

One standard method to analyze dynamics around $f_{\mathrm{S}}$ is to linearize the Vlasov equation (2) by expanding $f$ into

$$
f(q, p, t)=f_{\mathrm{S}}(p)+\epsilon g(q, p, t)
$$

The linearized Vlasov equation,

$$
\partial_{t} g+\left\{\mathcal{H}\left[f_{\mathrm{S}}\right], g\right\}+\left\{\mathcal{V}[g], f_{\mathrm{S}}\right\}=0
$$

gives the well-known Landau damping [32] of perturbation. If the Landau damping is strong enough, then the asymptotic state $f_{\mathrm{A}}$ may coincide with the initial stable stationary reference $f_{\mathrm{S}}$. On the other hand, if the Landau damping rate is close to zero, then nonlinear trapping [38] stops the damping, and the system relaxes to a different asymptotic state from $f_{\mathrm{S}}$. In the 
latter case, due to the nonlinearity, predicting the asymptotic state is nontrivial. The rearrangement formula is a powerful tool in the latter case as shown in this article.

The key idea of the rearrangement formula is as follows. Imagine that the initial state $f_{\mathrm{I}}$ asymptotically goes to a stationary state $f_{\mathrm{A}}$, which is still unknown. The asymptotic state constructs the asymptotic effective Hamiltonian $\mathcal{H}_{\mathrm{A}}=\mathcal{H}\left[f_{\mathrm{A}}\right]$ of the Vlasov equation, and the asymptotic Hamiltonian drives the system for a long time and takes it to the asymptotic state $f_{\mathrm{A}}$. Then, we check self-consistency between the imagined asymptotic state and the driven asymptotic state.

The above idea is theoretically formulated as follows. We decompose $f$ into another way as

$$
f(q, p, t)=f_{\mathrm{A}}(q, p)+\epsilon g_{\mathrm{T}}(q, p, t),
$$

where $f_{\mathrm{A}}$ and $\epsilon g_{\mathrm{T}}$ are respectively called the asymptotic (A) and transient $(\mathrm{T})$ parts. The A part $f_{\mathrm{A}}$ is picked up by use of a special case of the Abel's formula,

$$
f_{\mathrm{A}}(q, p)=\lim _{T \rightarrow \infty} \frac{1}{T} \int_{0}^{T} f(q, p, t) d t,
$$

and coincides with $\lim _{t \rightarrow \infty} f(q, p, t)$ if it exists. According to the A-T decomposition (10), the effective Hamiltonian is similarly decomposed as

$$
\mathcal{H}[f]=\mathcal{H}_{\mathrm{A}}+\epsilon \mathcal{V}_{\mathrm{T}},
$$

where the A part and the T part are defined by

$$
\mathcal{H}_{\mathrm{A}}=p^{2} / 2+\mathcal{V}\left[f_{\mathrm{A}}\right], \quad \mathcal{V}_{\mathrm{T}}=\mathcal{V}\left[g_{\mathrm{T}}\right],
$$

respectively. Substituting the decomposition (12) into the Vlasov equation (2), we have

$$
\partial_{t} f+\left\{\mathcal{H}_{\mathrm{A}}, f\right\}+\epsilon\left\{\mathcal{V}_{\mathrm{T}}, f\right\}=0 .
$$

If $f$ is always in an $O(\epsilon)$ neighborhood of $f_{\mathrm{I}}$, we can approximate the above exact equation as

$$
\partial_{t} f+\left\{\mathcal{H}_{\mathrm{A}}, f\right\}+\epsilon\left\{\mathcal{V}_{\mathrm{T}}, f_{\mathrm{I}}\right\}=0
$$

by omitting the $O\left(\epsilon^{2}\right)$ term which couples with the T part $\mathcal{V}_{\mathrm{T}}$. Nevertheless, we emphasize that the approximated equation (15) is not just a linearized equation like Eq. (9) for $O(\epsilon)$ terms, since $f$ includes the $O(1)$ term of $f_{\mathrm{A}}$. In other words, the term $\left\{\mathcal{H}_{\mathrm{A}}, f\right\}$ has nonlinearity. We remark that the criteria of truncation concerns to the surviving time scale of each term [27-29].

We can show that, under some assumptions, the unknown transient field $\mathcal{V}_{\mathrm{T}}$ appearing in the third term of the left-hand side of Eq. (15) does not contribute to determine the effective Hamiltonian $\mathcal{H}_{\mathrm{A}}$ [26]. Therefore, roughly speaking, the $\mathrm{A}$ part $f_{\mathrm{A}}$ is obtained as the asymptotic solution to the reduced equation

$$
\partial_{t} f+\left\{\mathcal{H}_{\mathrm{A}}, f\right\}=0 .
$$

Temporal evolution of $f$ is, hence, obtained as

$$
f(q, p, t)=f_{\mathrm{I}}\left(q_{-t}, p_{-t}\right),
$$

where $\left(q_{t}, p_{t}\right)$ is the orbit of the Hamiltonian dynamics governed by $\mathcal{H}_{\mathrm{A}}$ with the initial condition $(q, p)$. Abel's
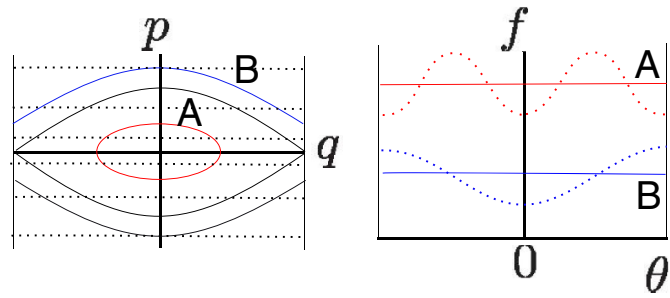

FIG. 1. (Color online) Schematic picture for the rearrangement formula (19) in the HMF model. The left panel shows $\mu$ space, and dotted and solid lines represent contours of $f_{\mathrm{I}}$ and $f_{\mathrm{A}}$ respectively. The right panel shows $\theta$ dependence of distribution functions, where the angle variable $\theta$ is defined by the asymptotic effective Hamiltonian $\mathcal{H}_{\mathrm{A}}$, and the red solid line marked by A corresponds to the red solid contour A of the left panel. Along the contour, the initial state $f_{\mathrm{I}}$ depends on the angle $\theta$ as described by the red dotted line in the right panel. The blue solid contour marked by B is another example. For the two examples $\mathrm{A}$ and $\mathrm{B}$, the positive $p$ axis corresponds to $\theta=0$. In this schematic picture $f_{\mathrm{I}}$ is assumed to be spatially homogeneous and a decreasing function of energy, though the rearrangement formula is also applicable to a spatially inhomogeneous $f_{\mathrm{I}}$.

formula (11) gives

$$
f_{\mathrm{A}}(q, p)=\lim _{T \rightarrow \infty} \frac{1}{T} \int_{0}^{T} f_{\mathrm{I}}\left(q_{-t}, p_{-t}\right) d t,
$$

and this is the time average of $f_{\mathrm{I}}$ along the orbit of the integrable Hamiltonian system $\mathcal{H}_{\mathrm{A}}$. Thus, introducing the angle-action variables $(\theta, J)$ associated with $\mathcal{H}_{\mathrm{A}}$, which is written as a function of $J$ only as $\mathcal{H}_{\mathrm{A}}(J)$, the ergodiclike formula replaces the time average of Eq. (18) with the iso- $J$ average

$$
f_{\mathrm{A}}=\frac{1}{2 \pi} \int_{-\pi}^{\pi} f_{\mathrm{I}}(q(\theta, J), p(\theta, J)) d \theta=\left\langle f_{\mathrm{I}}\right\rangle_{\mathcal{H}_{\mathrm{A}}} .
$$

This expression (19) is the rearrangement formula, which we will discuss. The concrete forms of $(\theta, J)$ and $\langle\bullet\rangle_{\mathcal{H}_{\mathrm{A}}}$ are exhibited in Appendix A with another equivalent practical expression of the average.

An illustrative presentation of the rearrangement formula (19) is to redistribute the height of the initial state $f_{\mathrm{I}}$ uniformly on each contour of the asymptotic Hamiltonian $\mathcal{H}_{\mathrm{A}}$ as described in Fig. 1. This procedure is consistent with the Jeans theorem [39] for constructing a stationary state, since the resulting state is constant on each contour of $\mathcal{H}_{\mathrm{A}}$. We note that neither the asymptotic state $f_{\mathrm{A}}$ nor Hamiltonian $\mathcal{H}_{\mathrm{A}}$ are still known, since both sides of the formula (19) depend on the asymptotic state. We have to determine the asymptotic state as it satisfies the self-consistent equation, and the determination will be done in Sec. III B for the HMF model.

It might be worth noting the similarity between the rearrangement formula and Lynden-Bell's theory. In the latter, we consider a waterbag initial state and divide the phase space into small phase space elements. Due to incompressibility of the Vlasov flow, the phase space elements are exclusive, and hence we redistribute them to phase space as maximizing the Fermi-Dirac like entropy with keeping the invariants of mass, momentum, and energy. In the former, we redistribute 
the phase space elements as Lynden-Bell's theory, but the redistribution is performed on each iso- $J$ contour.

\section{B. Application to the Hamiltonian mean-field model}

From symmetry of the system, we may assume that $\mathcal{M}_{y}=0$. We determine the asymptotic Hamiltonian without the external field,

$$
\mathcal{H}_{\mathrm{A}}=p^{2} / 2-M_{\mathrm{A}} \cos q,
$$

by solving the self-consistent equation

$$
M_{\mathrm{A}}=\iint \cos q\left\langle f_{\mathrm{I}}\right\rangle_{\mathcal{H}_{\mathrm{A}}} d q d p=\iint\langle\cos q\rangle_{\mathcal{H}_{\mathrm{A}}} f_{\mathrm{I}} d q d p .
$$

In the last equality we used the fact that $d q d p=d \theta d J$. The self-consistent equation (21) always has the solution of $M_{\mathrm{A}}=$ 0 corresponding to the strong Landau damping case, but we skip this trivial solution. The nonzero solution of $M_{\mathrm{A}}$, solved numerically, is the theoretical prediction to be examined in Sec. IV. Before going to numerical tests, we observe theoretically obtained approximate solutions to the self-consistent equation by expanding the self-consistent equation (21) with respect to small $M_{\mathrm{A}}$ [26]. The expansion leads to

$$
A\left[f_{\mathrm{I}}\right] M_{\mathrm{A}}^{1 / 2}+D\left[f_{\mathrm{I}}\right] M_{\mathrm{A}}+B\left[f_{\mathrm{I}}\right] M_{\mathrm{A}}^{3 / 2}=O\left(M_{\mathrm{A}}^{7 / 4}\right),
$$

where the functional $D$ is defined as

$$
D[f]=1+\frac{1}{2} \iint \frac{\partial_{p} f(q, p)}{p} d q d p
$$

which coincides with the dispersion function with zero frequency when $f$ does not depend on $q$. For simplicity again, we assume that the initial perturbation $g_{\mathrm{I}}=g(q, p, 0)$ is even with respect to both $q$ and $p$, and can be expanded into the Fourier series as

$$
g_{\mathrm{I}}(q, p)=\sum_{n \geqslant 1} \tilde{g}_{\mathrm{I}}(n, p) \cos (n q) .
$$

We note that, in this case, the contribution of the transient field $\mathcal{V}_{\mathrm{T}}$ to the asymptotic state is shown to vanish without approximation (16). See Appendix B for details. Then, the functional $D\left[f_{\mathrm{I}}\right]$ is reduced to $D\left[f_{\mathrm{S}}\right]$, and the functionals $A$ and $B$ are expressed by

$$
A\left[f_{\mathrm{I}}\right]=-\sum_{n \geqslant 1} \tilde{g}_{\mathrm{I}}(n, 0) C_{n}
$$

and

$$
B\left[f_{\mathrm{I}}\right]=-f_{\mathrm{S}}^{\prime \prime}(0) C_{1}+O\left(\epsilon M_{\mathrm{A}}^{3 / 2}\right)
$$

where

$$
C_{n}=M_{\mathrm{A}}^{-1 / 2} \iint \cos (n q)\langle\cos q\rangle_{\mathcal{H}_{\mathrm{A}}} d q d p .
$$

We remark that $C_{n}$ does not depend on $M_{\mathrm{A}}$ due to the scaling of $\langle\cos q\rangle_{\mathcal{H}_{\mathrm{A}}}$, and the constant values are numerically obtained as

$$
C_{0}=0, \quad C_{1} \simeq 5.169, \quad C_{2} \simeq 0.5360, \quad C_{3} \simeq-0.1043 .
$$

Neglecting $O\left(M_{\mathrm{A}}^{7 / 4}\right)$ terms in Eq. (22), we have the solutions as

$$
\sqrt{M_{\mathrm{A}}}=0, \frac{\sqrt{D^{2}-4 A B}-D}{2 B},
$$

where the second solution exists if and only if it is nonnegative.

\section{NUMERICAL TESTS OF THE REARRANGEMENT FORMULA}

\section{A. Two conditions to be tested}

The rearrangement formula (19) predicts the asymptotic value of order parameter $M_{\mathrm{A}}$ as the solutions to the selfconsistent equation (21), or as approximation (29). The zero solution corresponds to the strong Landau damping case, and hence the nonzero solution, in which we are interested, might be realized with the no Landau damping condition. However, validity of this expectation is still not clear since the rearrangement formula was successfully tested for initial waterbag states satisfying the generalized virial condition [22-24]. Then, we will make competition between the no Landau damping condition and a virial condition in Sec. IV B, and will clarify that the former is more relevant in our setting.

The other condition comes from the Casimir invariants of the Vlasov equation (2), where the invariants are functionals of the form

$$
S[f]=\iint s(f) d q d p, \quad s: C^{1} \text { function. }
$$

The rearrangement formula keeps all the Casimirs up to the linear order. This fact is shown from the expansion

$S\left[f_{\mathrm{I}}\right]-S\left[f_{\mathrm{A}}\right]=\iint s^{\prime}\left(f_{\mathrm{A}}\right) \delta f d \theta d J+O\left((\delta f)^{2}\right)=O\left((\delta f)^{2}\right)$,

where $\delta f=f_{\mathrm{I}}-f_{\mathrm{A}}$. The part of $s^{\prime}\left(f_{\mathrm{A}}\right)$ depends on $J$ only, and $\langle\delta f\rangle_{\mathcal{H}_{\mathrm{A}}}=0$ from $\left\langle f_{\mathrm{I}}\right\rangle_{\mathcal{H}_{\mathrm{A}}}=f_{\mathrm{A}}$. We again note that the angle-action variables $(\theta, J)$ associate with the asymptotic Hamiltonian $\mathcal{H}_{\mathrm{A}}$. The invariance, however, does not hold in higher orders. Indeed, for the Casimir

$$
S_{2}[f]=\iint f^{2} d q d p=\iint f^{2} d \theta d J,
$$

we have the discrepancy as

$$
S_{2}\left[f_{\mathrm{I}}\right]-S_{2}\left[f_{\mathrm{A}}\right]=\iint\left\langle(\delta f)^{2}\right\rangle_{\mathcal{H}_{\mathrm{A}}} d q d p,
$$

which is not zero in general. Therefore, the rearrangement formula may prefer initial states with which the Casimir is not greatly modified.

The above two conditions are examined by performing systematic numerical simulations of the Vlasov equation (2). We use the second-order semi-Lagrangian scheme [40] with the cubic $B$ spline interpolations in each step. Throughout this article we use the truncated single-particle phase space $(-\pi, \pi] \times[-4,4]$ and the time slice $\Delta t=0.05$. Asymptotic values are computed by taking averages over the time interval $[500,1000]$ if no comment appears. The phase space is divided into the grid of size $G \times G$, which is called the grid size $G$. 
In this section, we consider the HMF model without external field as in the previous section, and observe $M_{x}=\mathcal{M}_{x}[f]$.

\section{B. The no Landau damping condition}

The generalized virial condition represents quasistationarity of a given waterbag initial state [18], and it is not straightforward to apply it for other initial states. On the other hand, the proper virial condition is not useful for spatially periodic systems. Thus, for making competition with the no Landau damping condition, we introduce another type of virial condition with keeping the meaning of quasistationarity.

The proper virial condition is derived by differentiating $P(t)=\sum_{j=1}^{N} p_{j} q_{j} / N$ and taking the long-time average. The periodic boundary condition of the HMF model suggests to consider $Q(t)=\sum_{j=1}^{N} p_{j} \varphi\left(q_{j}\right) / N$, where $\varphi$ is an arbitrary smooth periodic function. Taking the limit $N \rightarrow \infty$, we replace the arithmetic mean with the average over the distribution function $f$. If $f_{\mathrm{I}}$ is stationary, we have the relation

$$
\left\langle p^{2} \frac{d \varphi}{d q}(q)-\varphi(q) \frac{d \mathcal{V}\left[f_{\mathrm{I}}\right]}{d q}(q)\right\rangle_{\mathrm{I}}=0,
$$

where $\langle\bullet\rangle_{\mathrm{I}}=\iint \bullet f_{\mathrm{I}} d q d p$. Hereafter we put $\varphi(q)=\sin q$ which gives

$$
\left\langle p^{2} \cos q-\sin q \frac{d \mathcal{V}\left[f_{\mathrm{I}}\right]}{d q}(q)\right\rangle_{\mathrm{I}}=0 .
$$

We refer to Eq. (35) as the periodic virial condition. We note that the above condition is equivalent with $\ddot{M}_{x}(0)=0$, and $\dot{M}_{x}(0)=0$ is also satisfied for even $f_{\mathrm{I}}$ with respect to $p$. These vanishing derivatives imply that the periodic virial condition represents quasistationarity in a short-time interval. The condition (35) will be compared with the no Landau damping condition, which is explicitly written as

$$
D\left[f_{\mathrm{S}}\right]=0
$$

since positive, negative, and vanishing $D$ imply that $f_{\mathrm{S}}$ is stable, unstable, and marginal respectively.

For the competition, we prepare a family of initial states as

$$
f_{\mathrm{I}}\left(q, p ; T_{0}, T_{1}\right)=f_{\mathrm{MB}}\left(p ; T_{0}\right)+\epsilon f_{\mathrm{MB}}\left(p ; T_{1}\right) \cos q,
$$

where $f_{\mathrm{MB}}$ denotes the Maxwell-Boltzmann distribution

$$
f_{\mathrm{MB}}(p ; T)=\frac{1}{2 \pi \sqrt{2 \pi T}} e^{-p^{2} / 2 T} .
$$

The unperturbed part gives

$$
D\left[f_{\mathrm{MB}}\right]=1-\frac{1}{2 T_{0}},
$$

and the no Landau damping condition $D=0$ is realized at the critical temperature $T_{0}=T_{\mathrm{c}}=1 / 2$ of the second order phase transition $[35,36]$. The Maxwell-Boltzmann distribution is stable for $T>T_{\mathrm{c}}$. On the other hand, the periodic virial condition (35) is realized at $T_{1}=T_{\mathrm{c}}=1 / 2$. The family (37), therefore, can exclusively satisfy one of the two conditions as follows:

Case 1. $f_{\mathrm{I}}\left(q, p ; T_{\mathrm{c}}, T\right)$ with $T>T_{\mathrm{c}}$ : The unperturbed term $f_{\mathrm{MB}}\left(p ; T_{\mathrm{c}}\right)$ satisfies the no Landau damping condition (36), but $f_{\mathrm{I}}$ breaks the periodic virial condition (35).

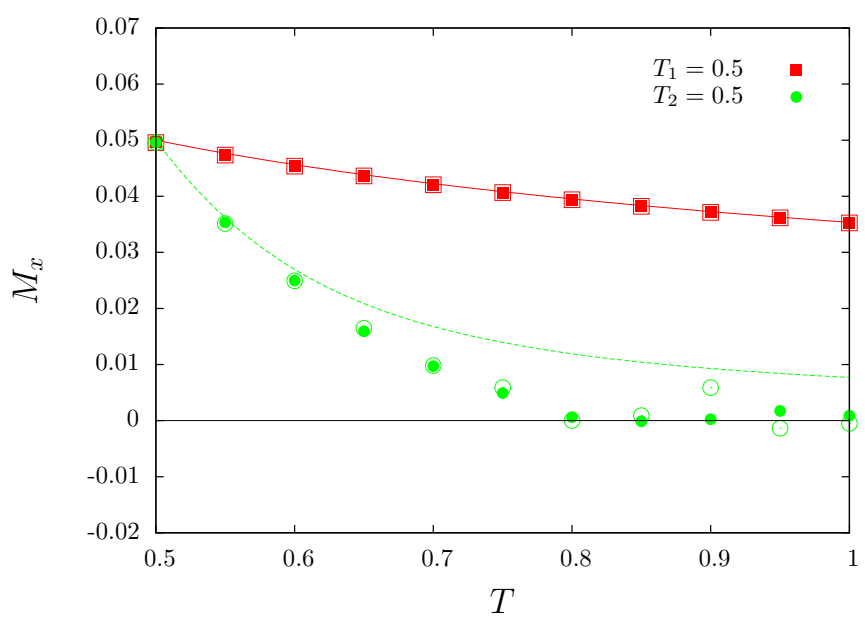

FIG. 2. (Color online) Asymptotic values of $M_{x}$ for the initial distributions (37). The red squares are for Case 1, and the green circles are for Case 2. The open and filled symbols are computed with the grid sizes $G=256$ and 512 respectively. $\epsilon=0.1$. The red solid and the green dashed lines are from the approximated theory (29) for Case 1 and Case 2 respectively.

Case 2. $f_{\mathrm{I}}\left(q, p ; T, T_{\mathrm{c}}\right)$ with $T>T_{\mathrm{c}}: f_{\mathrm{I}}$ satisfies the periodic virial condition (35), but the unperturbed term $f_{\mathrm{MB}}(p ; T)$ breaks the no Landau damping condition (36).

As shown in Fig. 2, the rearrangement formula gives precise prediction in Case 1 for all $T>T_{\mathrm{c}}$ and in Case 2 for $T$ close to $T_{\mathrm{c}}$. In Case 2, the agreement between the rearrangement formula and numerics becomes worse as $T$ increases, that is, the Landau damping rate gets larger, though the periodic virial condition holds. From the numerical observation, we conclude that the no Landau damping condition is more relevant than the periodic virial condition for the perturbed Maxwell-Boltzmann states (37).

\section{Casimir invariance}

The previous work [26] uses the initial perturbation $g_{\text {I }}$ having only the first Fourier mode with respect to the position $q$ [see Eq. (24)], and shows that the rearrangement formula gives precise predictions at the critical point even for rather large perturbation. However, any Fourier modes can contribute to the asymptotic value of order parameter $M_{\mathrm{A}}$ through mode couplings. We will reveal that initial perturbation is also restrictive by adding the second Fourier mode to it, and will qualitatively explain discrepancy between the theory and numerics from the viewpoint of the Casimir invariance.

We prepare the initial state as

$$
f_{\mathrm{I}}(q, p)=f_{\mathrm{MB}}\left(p ; T_{\mathrm{c}}\right) \sum_{k=0}^{2} \epsilon_{k} \cos k q,
$$

where $\epsilon_{0}=1$. We call the term $\epsilon_{k} \cos k q$ the $k$ th mode. We set the stationary state as $f_{\mathrm{S}}(p)=f_{\mathrm{MB}}\left(p ; T_{\mathrm{c}}\right)$ for an independent test of the no Landau damping condition. The first mode is included to escape from $M_{x}=0$.

For a fixed value of $\epsilon_{1}=0.1$, we show $\epsilon_{2}$ dependence of $M_{x}$ in Fig. 3. The theoretical prediction is in good agreement with numerics for small $\left|\epsilon_{2}\right|$, but discrepancy tends to grow 


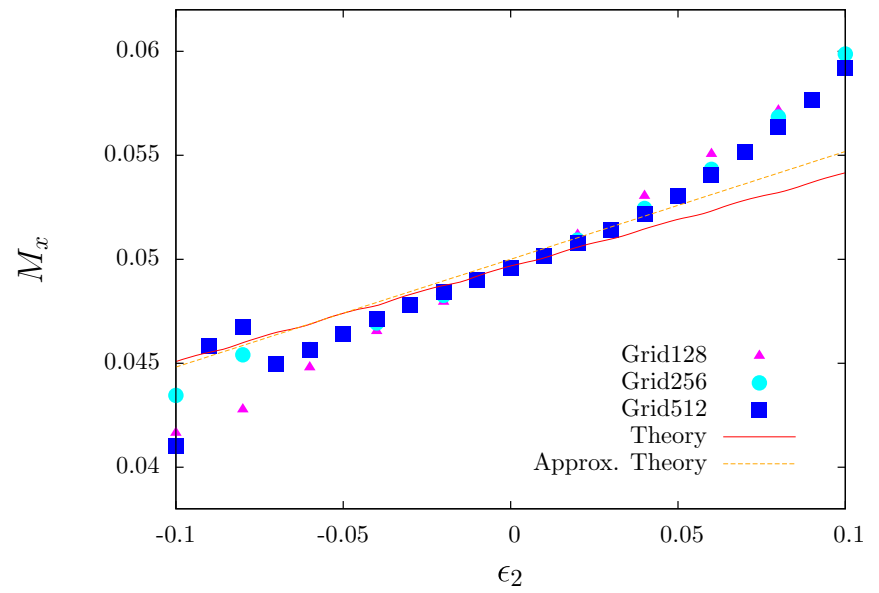

FIG. 3. (Color online) $\epsilon_{2}$ dependence of $M_{x}$ with the fixed $\epsilon_{1}=$ 0.1 for the initial state (40). The red solid line represents the full theory (21), the orange dashed line is approximated theory (29), and points are from numerics. The sizes of grid are $G=128$ (purple triangles), 256 (light blue circles), and 512 (blue squares).

for large $\left|\epsilon_{2}\right|$. Moreover, in the large $\epsilon_{2}$ region, the theoretical prediction is smaller than numerics, while the Landau damping mechanism provides the inverse result. Existence of the second mode is, therefore, an independent mechanism to yield discrepancy between the theory and numerics. We remark that there is a nonregular dependence on $\epsilon_{2}$ around $\epsilon_{2} \simeq-0.08$, but the mechanism of this dependence is not clear yet.

Looking at Fig. 3, we expect that $\left|\epsilon_{2}\right|$ must be much smaller than $\left|\epsilon_{1}\right|$. The above expectation is confirmed by varying $\epsilon_{1}$ for a fixed value of $\epsilon_{2}=0.01$. Values of the asymptotic magnetization $M_{x}$ are reported in Fig. 4 with the relative error defined by

$$
R=\frac{M_{\text {numerics }}-M_{\text {theory }}}{M_{\text {theory }}},
$$

where $M_{\text {theory }}$ and $M_{\text {numerics }}$ are respectively obtained theoretically (21) and numerically. In the large $\epsilon_{1}$ region, the minus of relative error grows as $\epsilon_{1}$ gets large. This growth of the relative error of $O\left(\epsilon_{1}^{2}\right)$ might be rather natural since we omitted $O\left(\epsilon^{2}\right)$ terms in Eq. (15). Interesting observations are that the relative error changes the sign around the minimum point, and grows even as $\epsilon_{1}$ decreases.

Let us discuss the mechanism of the $\epsilon_{1}$ dependence of the relative error from the viewpoint of invariance of the Casimir $S_{2}$ (32). The initial value of $S_{2}$ is computed as

$$
S_{2}\left[f_{\mathrm{I}}\right]=\iint f_{\mathrm{I}}(q, p)^{2} d q d p=\frac{1}{4 \pi \sqrt{\pi T_{\mathrm{c}}}}\left(1+\frac{\epsilon_{1}^{2}}{2}+\frac{\epsilon_{2}^{2}}{2}\right) .
$$

On the other hand, under some phenomenological assumptions, we approximate $S_{2}\left[f_{\mathrm{A}}\right]$ as

$$
S_{2}\left[f_{\mathrm{A}}\right] \simeq \frac{1}{4 \pi \sqrt{\pi T_{\mathrm{c}}}}\left(1+\frac{\epsilon_{1}^{2}}{2}+2 c_{2} \epsilon_{1} \epsilon_{2}+c_{2}^{2} \epsilon_{2}^{2}\right),
$$

where $c_{2}=C_{2} / C_{1} \simeq 0.1$ from the values of $C_{1}$ and $C_{2}$, (28). See Appendix $C$ for deriving the approximation (43). Comparing the asymptotic value (43) with the initial value

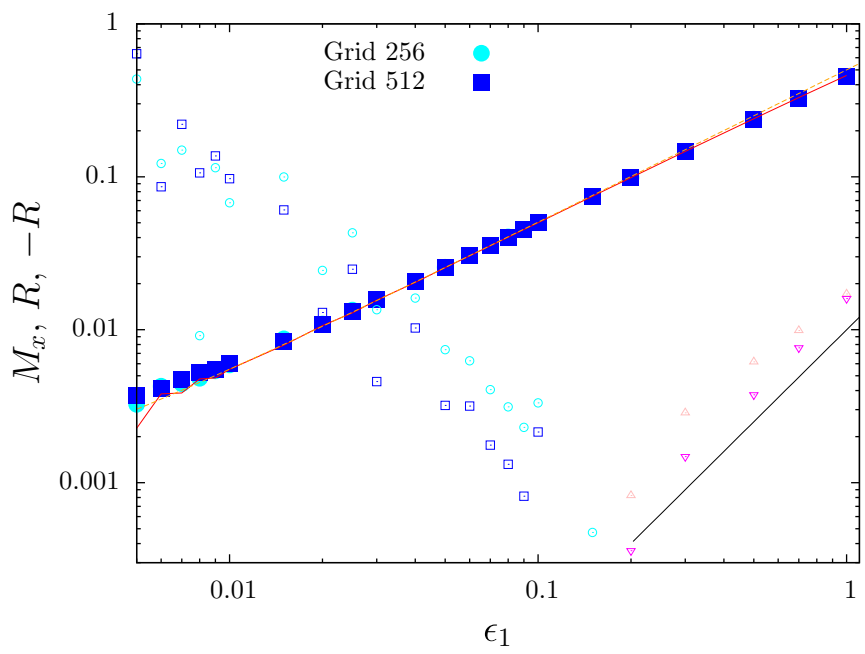

FIG. 4. (Color online) $\epsilon_{1}$ dependence of $M_{x}$ with the fixed $\epsilon_{2}=$ 0.01 for the initial state (40). The solid red line represents the full theory (21), the orange dashed line is the approximated theory (29), and filled points are from numerics. The grid sizes are $G=256$ (light blue circles) and 512 (blue squares). Open symbols represent relative errors with the full theory: $R$ for $G=256$ (light blue circles) and 512 (blue squares), and $-R$ for $G=256$ (pink triangles) and 512 (purple inverse triangles). The black solid line is a guide for eyes, and has slope 2 .

(42), we find that invariance of the Casimir $S_{2}$ is realized for $\epsilon_{2}$ satisfying

$$
\epsilon_{2}^{2}=4 c_{2} \epsilon_{1} \epsilon_{2}+2 c_{2}^{2} \epsilon_{2}^{2}
$$

which is approximately solved by

$$
\epsilon_{2} \simeq 4 c_{2} \epsilon_{1} \simeq 0.4 \epsilon_{1} \text {. }
$$

The relation (45) qualitatively explains the minimum point of the relative error in Fig. 4.

The estimation of $S_{2}\left[f_{\mathrm{A}}\right]$, (43), also qualitatively explains underestimation by the theory for large $\epsilon_{2} / \epsilon_{1}$. Suppose $\epsilon_{2} \simeq$ $\epsilon_{1}$. In this case $S_{2}\left[f_{\mathrm{I}}\right]$ is larger than $S_{2}\left[f_{\mathrm{A}}\right]$ due to the factor $c_{2}=C_{2} / C_{1} \simeq 0.1$. In the full Vlasov system, $S_{2}$ is conserved and hence the lost part $S_{2}\left[f_{\mathrm{I}}\right]-S_{2}\left[f_{\mathrm{A}}\right]$ must be covered by, for instance, increasing amplitude of the first Fourier mode relating to $M_{\mathrm{A}}$. Then, underestimation by the theory possibly occurs.

\section{RESPONSE THEORY TO THE EXTERNAL FIELD}

We have dealt with the HMF model without external field in Secs. III and IV. In this section, we consider the response to the nonzero external field and the critical exponents $\gamma_{ \pm}$and $\delta$ defined as

$$
M_{\mathrm{A}}-M_{\mathrm{I}} \propto\left|T-T_{\mathrm{c}}\right|^{-\gamma_{ \pm}} h, \quad M_{\mathrm{A}} \propto h^{1 / \delta}
$$

in the limit of $h \rightarrow 0$, where $h$ is the strength of the external field, $M_{\mathrm{I}}$ is the initial order parameter, and $\gamma_{+}$and $\gamma_{-}$are defined in high- and low-temperature sides respectively. We note that the considering family of states may be thermal equilibrium states or QSSs, and a certain parameter plays the role of temperature in the latter QSS case. The rearrangement formula gives $\gamma_{+}=1$ and the nonclassical exponents of 
$\gamma_{-}=1 / 4$ [33] and $\delta=3 / 2$ [26] in a wide class of onedimensional Vlasov dynamics with the periodic boundary condition [34], while statistical mechanics gives $\gamma_{+}=\gamma_{-}=1$ and $\delta=3$.

We numerically found that the rearrangement formula requires two conditions to be satisfied: One is the no Landau damping condition and the other comes from the Casimir invariance. These conditions restrict applicable initial states for the rearrangement formula. However, the conditions reinforce validity of use of the rearrangement formula as the response theory to the external field, which turns on at the initial time and goes to be constant asymptotically, since this setting satisfies both the conditions as discussed in the following.

Performing the Laplace transform of the external field we get a pole at the origin of the Laplace space (the complex frequency plane), and the pole provides the asymptotically surviving response [41,42]. We hence conjecture that this pole effectively restores the no Landau damping condition even if the unperturbed stationary state breaks the condition. Moreover, for the critical exponents, the interesting reference states are close to the critical state which satisfies the no Landau damping condition.

It is not hard to see that the second condition, the Casimir invariance, is satisfied for small $h$. As shown in Eq. (31), invariance of all the Casimirs is satisfied by the rearrangement theory up to the linear order. The critical exponents are defined in the limit of small external field, and hence the Casimir invariance is not an obstacle for computing them.

\section{SUMMARY AND DISCUSSIONS}

We discussed conditions to use the rearrangement formula around spatially homogeneous stable stationary states in the HMF model, and numerically derived two conditions: One is for the stable stationary reference state, and the other is for the whole initial state. The former is the no Landau damping condition, which was previously suggested [26]. We compared this condition with a virial condition, which we called the periodic virial condition, and numerically clarified that the no Landau damping condition is more crucial than the periodic virial condition. The latter comes from the Casimir invariance: The theory prefers initial perturbed states which keep the Casimirs well. Breaking the former and the latter, theoretical predictions tend to overestimate and underestimate respectively, and hence we may conclude that the two conditions are independent.

Due to the conditions, the rearrangement formula is restrictive for using as a nonequilibrium statistical mechanics. Nevertheless, the theory is useful as a response theory to the external field saturating to a small constant asymptotically, since the conditions are satisfied in such a situation. In particular, the conditions validate to compute the critical exponents in the use of the rearrangement formula.

Another important benefit of the present work is that the conditions suggest a direction for improving the rearrangement formula: The theory could be improved by inputting the Landau damping and the Casimir invariants. For instance, we expect that nonlinear trapping plays an important role to form a magnetized asymptotic state, and an improved theory may be derived by considering the competition between the linear
Landau damping and the nonlinear trapping as discussed for forming traveling small clusters [37]. Such an improvement is interesting and worthwhile for constructing a nonequilibrium statistical mechanics, but remains as a future work.

A similar formula with the rearrangement formula has been also derived by de Buyl et al. [43] for a small system of $O(\epsilon)$ contacting with a huge bath of $O(1)$ through long-range interactions. In this setting, magnetization in the huge bath plays the role of external field for the system, and the rearrangement formula possibly provides good predictions as discussed in the present article, if the bath is huge enough and static accordingly. We remark that the system is driven by the bath magnetization only, and no self-consistent condition is needed for the system magnetization, since the latter is small enough and can be omitted.

This article dealt with the HMF model only, but, from the physical mechanism leading the two conditions, one may expect that generic systems having long-range interactions share the two conditions. Examinations for several systems remain to be done. We discussed initial states around stable stationary states, but the rearrangement formula has been successfully used in three-dimensional self-gravitating systems with waterbag initial conditions, which satisfy the virial condition [25]. It also remains to reveal a relation between the two types of initial states, which are perturbed stable stationary states and waterbag states.

We end this article by mentioning the discussion on parametric resonance for initial states which are neither perturbed stable stationary states nor waterbag states satisfying the virial condition [25]. We have discussed the discrepancy induced with the higher Fourier modes based on the Casimir invariants. On the other hand, there is another explanation based on the parametric resonance induced by the higher moments [25]. Clarifying the relation between the two explanations remains for a future work.

\section{ACKNOWLEDGMENTS}

Y.Y.Y. acknowledges the support of JSPS KAKENHI Grant No. 23560069. S.O. acknowledges the support of Grant-in-Aid for JSPS Fellows Grant No. 254728.

\section{APPENDIX A: EXPLICIT FORM OF $\langle\bullet\rangle_{\mathcal{H}_{\mathrm{A}}}$}

The angle-action variables $(\theta, J)$ are obtained by

$$
\theta=\frac{\partial W}{\partial J}(q, J), \quad J=\frac{1}{2 \pi} \oint p d q,
$$

where the integral is performed along a periodic orbit, and the generating function $W$ is

$$
W(q, J)=\int_{0}^{q} p\left(q^{\prime}, J\right) d q^{\prime} .
$$

To express these variables for the asymptotic effective Hamiltonian system

$$
\mathcal{H}_{\mathrm{A}}=\frac{p^{2}}{2}-M_{\mathrm{A}} \cos q
$$


we use the variable $k$ defined as

$$
k \equiv \sqrt{\frac{\mathcal{H}_{\mathrm{A}}+M_{\mathrm{A}}}{2 M_{\mathrm{A}}}},
$$

and the Legendre elliptic integrals of the first and the second kinds respectively defined by

$$
\begin{aligned}
& F(\phi, k)=\int_{0}^{\phi} \frac{d x}{\sqrt{1-k^{2} \sin ^{2} x}}, \\
& E(\phi, k)=\int_{0}^{\phi} \sqrt{1-k^{2} \sin ^{2} x} d x .
\end{aligned}
$$

These integrals induce the complete elliptic integrals of the first and the second kinds respectively defined as

$$
K(k)=F(\pi / 2, k), \quad E(k)=E(\pi / 2, k) .
$$

The action and the angle variables are then expressed in the forms

$$
J= \begin{cases}\frac{8 \sqrt{M_{\mathrm{A}}}}{\pi}\left[E(k)-\left(1-k^{2}\right) K(k)\right], & k<1, \\ \frac{4 \sqrt{M_{\mathrm{A}}} k}{\pi} E(1 / k), & k>1,\end{cases}
$$

and

$$
\theta= \begin{cases}\frac{\pi}{2} F(Q, k) / K(k), & p \geqslant 0, k<1, \\ \frac{\pi}{2}[2-F(Q, k) / K(k)], & p<0, k<1, \\ \pi \operatorname{sgn}(p) F(Q, 1 / k) / K(1 / k), & k>1,\end{cases}
$$

where $Q$ is defined as $k \sin Q=\sin (q / 2)$ for $k<1$ and as $Q=q / 2$ for $k>1$. See Ref. [44] for details.

Using the variable transforms from $\theta$ to $Q$, for an observable $B(q, p)$ even with respect to $p$, we can write the average of $B(q, p)$ over an iso- $\mathcal{H}_{\mathrm{A}}$ curve as

$$
\langle B\rangle_{\mathcal{H}_{\mathrm{A}}}= \begin{cases}\frac{1}{2 K(k)} \int_{-\pi / 2}^{\pi / 2} \frac{B(q, p)}{\sqrt{1-k^{2} \sin ^{2} Q}} d Q, & k<1, \\ \frac{1}{2 K(1 / k)} \int_{-\pi / 2}^{\pi / 2} \frac{B(q, p)}{\sqrt{1-k^{-2} \sin ^{2} Q}} d Q, & k>1,\end{cases}
$$

where $B(q, p)$ must be transformed to a function of $(Q, k)$. The average $\langle B\rangle_{\mathcal{H}_{\mathrm{A}}}$ is obtained as a function of $k$.

For a Hamiltonian system $\mathcal{H}(q, p)$, there is another expression of the average over isoenergy curves as

$$
\langle B\rangle_{\delta}=\frac{\iint \delta(\mathcal{H}(q, p)-E) B(q, p) d q d p}{\iint \delta(\mathcal{H}(q, p)-E) d q d p} .
$$

This expression has been applied to the HMF model [23,24] and to the three-dimensional self-gravitating system [25]. We can show the equality $\langle B\rangle_{H}=\langle B\rangle_{\delta}$ for the one-dimensional case in each region of phase space where we can construct the inverse function of the Hamiltonian $\mathcal{H}(J)$. Using the relation $d q d p=d \theta d J$, and the variable change $x=\mathcal{H}(J)$, we can modify $\langle B\rangle_{\delta}$ as

$$
\begin{aligned}
\langle B\rangle_{\delta} & =\frac{\iint \delta(x-E) B\left(\theta, \mathcal{H}^{-1}(x)\right) d \theta \frac{d x}{\Omega\left(\mathcal{H}^{-1}(x)\right)}}{\iint \delta(x-E) d \theta \frac{d x}{\Omega\left(\mathcal{H}^{-1}(x)\right)}} \\
& =\frac{1}{2 \pi} \int B\left(\theta, \mathcal{H}^{-1}(E)\right) d \theta=\langle B\rangle_{\mathcal{H}},
\end{aligned}
$$

where $\Omega(J)=(d \mathcal{H} / d J)(J)$, we used the fact that $\Omega(J)>0$ except for $J$ corresponding to the separatrix, and we denoted the observable $B$ as $B(\theta, J)$ even in the angle-action coordinate for simplicity of notation. The expression $\langle B\rangle_{\delta}$ might be useful when deriving the angle-action variables is hard.

\section{APPENDIX B: DERIVATION OF THE REARRANGEMENT FORMULA WITHOUT OMITTING THE TERM INCLUDING T FIELD $\mathcal{V}_{\mathrm{T}}$}

Precisely, the asymptotic part $f_{\mathrm{A}}$ is constructed by the two terms of so-called the O'Neil term $f_{\mathrm{O}}$ and the Landau term $f_{\mathrm{L}}$ defined by

$$
\begin{aligned}
& f_{\mathrm{O}}=e^{-t\left\{\mathcal{H}_{\mathrm{A}}, \bullet\right\}} f_{\mathrm{I}}, \\
& f_{\mathrm{L}}=-\int_{0}^{t} e^{-(t-s)\left\{\mathcal{H}_{\mathrm{A}}, \bullet\right\}}\left\{\mathcal{V}_{\mathrm{T}}(s), f_{\mathrm{I}}\right\} d s,
\end{aligned}
$$

respectively [28,29]. By use of them, the solution to the Tlinearized equation (15) indeed is written as $f_{\mathrm{TL}}(q, p, t)=$ $f_{\mathrm{O}}(q, p, t)+\epsilon f_{\mathrm{L}}(q, p, t)$. The O'Neil term gives the expression $\left\langle f_{\mathrm{I}}\right\rangle_{\mathcal{H}_{\mathrm{A}}}$ in the limit $t \rightarrow \infty$. The Landau term comes from the neglected term of $\epsilon\left\{\mathcal{V}_{\mathrm{T}}, f_{\mathrm{I}}\right\}$ [see Eq. (15)], and is neglected since it has no contribution to the asymptotic Hamiltonian $\mathcal{H}_{\mathrm{A}}$ [26]. Meanwhile, it has not been shown whether or not the contribution of the Landau term to the asymptotic distribution vanishes. We show that the Landau term completely vanishes in the limit of $t \rightarrow \infty$, when the initial state $f_{\mathrm{I}}(q, p)$ is even with respect to both $q$ and $p$, that is, $f_{\mathrm{I}}(q, p)=f_{\mathrm{I}}(-q, p)=$ $f_{\mathrm{I}}(-q,-p)=f_{\mathrm{I}}(q,-p)$. The initial conditions dealt with in this article have this symmetry.

Let us show $f(q, p, t)=f(-q,-p, t)$ for $t \geqslant 0$ if $f_{\mathrm{I}}(q, p)=f_{\mathrm{I}}(-q,-p)$ at initial. Changing variables by $(q, p) \mapsto(-q,-p)$, it is easy to show that $f(-q,-p, t)$ is also a solution to the Vlasov equation with the initial condition $f_{\mathrm{I}}(q, p)$. It is, then, shown that $f(q, p, t)=f(-q,-p, t)$, due to the existence and uniqueness of solution to the Vasov equation [9]. The fact $\mathcal{M}_{y}[f](t)=0$ is immediately shown, and it is reasonable to consider that the asymptotic Hamiltonian can be given in the form

$$
\mathcal{H}_{\mathrm{A}}(q, p)=p^{2} / 2-M_{\mathrm{A}} \cos q,
$$

which says $\mathcal{M}_{y}\left[f_{\mathrm{A}}\right]=0$. Thus, the definition of the transient part $g_{\mathrm{T}}$ [Eq. (10)], induces that $\mathcal{M}_{y}\left[g_{\mathrm{T}}\right](t)=0$. form

The asymptotic form of the Landau term is written in the

$$
\begin{aligned}
\lim _{t \rightarrow \infty} f_{\mathrm{L}}= & \left\langle\sin q \frac{\partial f_{\mathrm{I}}}{\partial p}\right\rangle_{\mathcal{H}_{\mathrm{A}}} \int_{0}^{\infty} \mathcal{M}_{x}\left[g_{\mathrm{T}}\right](t) d t \\
& -\left\langle\cos q \frac{\partial f_{\mathrm{I}}}{\partial p}\right\rangle_{\mathcal{H}_{\mathrm{A}}} \int_{0}^{\infty} \mathcal{M}_{y}\left[g_{\mathrm{T}}\right](t) d t .
\end{aligned}
$$

The symmetry for $q \rightarrow-q$ of $f_{\mathrm{I}}$ and of $\mathcal{H}_{\mathrm{A}}$ vanishes in the first term of the right-hand side, and the fact that $\mathcal{M}_{y}\left[g_{\mathrm{T}}\right](t)=0$ eliminates the second. We, therefore, conclude $\lim _{t \rightarrow \infty} f_{\mathrm{L}}=0$. This procedure can be applied to more general one-dimensional periodic systems than we have dealt with finite number of Fourier modes in Ref. [34].

\section{APPENDIX C: ASYMPTOTIC VALUE OF THE CASIMIR $\boldsymbol{S}_{2}$}

The Jeans theorem [39] states that a state is stationary if and only if it depends on $(q, p)$ solely through the first integrals, in 
our case, the effective Hamiltonian. The asymptotic stationary state is, therefore, expressed as

$$
f_{\mathrm{A}}(q, p)=F_{\mathrm{A}}\left(p^{2} / 2-M_{\mathrm{A}} \cos q\right) .
$$

Now $M_{\mathrm{A}}$ is assumed to be small and we further assume that $F_{\mathrm{A}}$ accepts the Taylor expansion

$$
\begin{aligned}
F_{\mathrm{A}}\left(p^{2} / 2-M_{\mathrm{A}} \cos q\right)= & F_{\mathrm{A}}\left(p^{2} / 2\right)-M_{\mathrm{A}} \cos q F_{\mathrm{A}}^{\prime}\left(p^{2} / 2\right) \\
& +\frac{M_{\mathrm{A}}^{2}}{2} \cos ^{2} q F_{\mathrm{A}}^{\prime \prime}\left(p^{2} / 2\right)+\cdots .
\end{aligned}
$$

The validity of this assumption is not obvious due to the averaging procedure $f_{\mathrm{A}}=\left\langle f_{\mathrm{I}}\right\rangle_{\mathcal{H}_{\mathrm{A}}}$ which makes a cusp for the function $\langle\cos q\rangle_{\mathcal{H}_{\mathrm{A}}}$ at the separatrix energy [26] for instance. However, it helps us to discuss the Casimir $S_{2}$ qualitatively.

We assume that $F_{\mathrm{A}}\left(p^{2} / 2\right)$ is close to the unperturbed Maxwell-Boltzmann distribution $f_{\mathrm{MB}}\left(p ; T_{\mathrm{c}}\right)=F_{\mathrm{MB}}\left(p^{2} / 2\right)$, where $F_{\mathrm{MB}}(E) \propto e^{-E / T_{\mathrm{c}}}$, and write it as

$$
F_{\mathrm{A}}\left(p^{2} / 2\right)=F_{\mathrm{MB}}\left(p^{2} / 2\right)+\epsilon G\left(p^{2} / 2\right)
$$

with a small parameter $\epsilon$ of $O\left(\epsilon_{1}\right)$ or $O\left(\epsilon_{2}\right)$. Remembering that $M_{\mathrm{A}}$ is of $O(\epsilon)$, omitting $O\left(\epsilon^{3}\right)$ and using $F_{\mathrm{MB}}^{\prime}=-F_{\mathrm{MB}} / T_{\mathrm{c}}$, we have

$$
\begin{aligned}
f_{\mathrm{A}} \simeq & \left(1+\frac{M_{\mathrm{A}}^{2}}{4 T_{\mathrm{c}}^{2}}\right) F_{\mathrm{MB}}+\epsilon G+\left(\frac{M_{\mathrm{A}}}{T_{\mathrm{c}}} F_{\mathrm{MB}}-\epsilon M_{\mathrm{A}} G^{\prime}\right) \cos q \\
& +\frac{M_{\mathrm{A}}^{2}}{4 T_{\mathrm{c}}^{2}} F_{\mathrm{MB}} \cos 2 q .
\end{aligned}
$$

Straightforward computations give

$$
\begin{aligned}
S_{2}\left[f_{\mathrm{A}}\right] \simeq & \frac{1}{4 \pi \sqrt{\pi T_{\mathrm{c}}}}\left(1+\frac{M_{\mathrm{A}}^{2}}{T_{\mathrm{c}}^{2}}+2 \epsilon \frac{\int F_{\mathrm{MB}} G d p}{\int F_{\mathrm{MB}}^{2} d p}\right. \\
& \left.+\epsilon^{2} \frac{\int G^{2} d p}{\int F_{\mathrm{MB}}^{2} d p}\right)
\end{aligned}
$$

by omitting $O\left(\epsilon^{3}\right)$. Introducing $c_{2}=C_{2} / C_{1}$ and remembering that

$$
M_{\mathrm{A}}=-\frac{A}{B}=T_{\mathrm{c}}\left(\epsilon_{1}+c_{2} \epsilon_{2}+\cdots\right)
$$

at the critical point $T_{\mathrm{c}}$ from Eqs. (25), (26), and (29), the asymptotic value is rewritten as

$$
\begin{aligned}
S_{2}\left[f_{\mathrm{A}}\right] \simeq & \frac{1}{4 \pi \sqrt{\pi T_{\mathrm{c}}}}\left(1+\frac{\left(\epsilon_{1}+c_{2} \epsilon_{2}\right)^{2}}{2}\right)+\frac{1}{4 \pi \sqrt{\pi T_{\mathrm{c}}}} \\
& \times\left(\frac{\left(\epsilon_{1}+c_{2} \epsilon_{2}\right)^{2}}{2}+2 \epsilon \frac{\int F_{\mathrm{MB}} G d p}{\int F_{\mathrm{MB}}^{2} d p}+\epsilon^{2} \frac{\int G^{2} d p}{\int F_{\mathrm{MB}}^{2} d p}\right) .
\end{aligned}
$$

It has been numerically reported that the rearrangement formula gives precise predictions for $\epsilon_{2}=0$ [26]. Thus, comparing (C7) with (42), we assume that the equality

$$
\epsilon_{1}^{2}+4 \epsilon \frac{\int F_{\mathrm{MB}} G d p}{\int F_{\mathrm{MB}}^{2} d p}+2 \epsilon^{2} \frac{\int G^{2} d p}{\int F_{\mathrm{MB}}^{2} d p}=O\left(\epsilon^{3}\right)
$$

holds for $\epsilon_{2}=0$ and for small $\epsilon_{2}$. This assumption induces the asymptotic value of Eq. (43).
[1] A. Campa, T. Dauxois, D. Fanelli, and S. Ruffo, Physics of LongRange Interacting Systems (Oxford University Press, Oxford, 2014).

[2] A. Campa, T. Dauxois, and S. Ruffo, Statistical mechanics and dynamics of solvable models with long-range interactions, Phys. Rep. 480, 57 (2009).

[3] J. Binney and S. Tremaine, Galactic Dynamics, 2nd ed. (Princeton University Press, Princeton, NJ, 2008).

[4] D. H. Zanette and M. A. Montemurro, Dynamics and nonequilibrium states in the Hamiltonian mean-field model: A. closer look, Phys. Rev. E 67, 031105 (2003).

[5] Y. Y. Yamaguchi, J. Barré, F. Bouchet, T. Dauxois, and S. Ruffo, Stability criteria of the Vlasov equation and quasi-stationary states of the HMF model, Physica A 337, 36 (2004).

[6] M. Kastner, Diverging Equilibration Times in Long-Range Quantum Spin Models, Phys. Rev. Lett. 106, 130601 (2011).

[7] W. Braun and K. Hepp, The Vlasov dynamics and its fluctuations in the $1 / N$ limit of interacting classical particles, Commun. Math. Phys. 56, 101 (1977).

[8] R. L. Dobrushin, Vlasov equations, Funct. Anal. Appl. 13, 115 (1979).

[9] H. Spohn, Large Scale Dynamics of Interacting Particles (Springer-Verlag, Heidelberg, 1991).

[10] K. S. Fine, A. C. Cass, W. G. Flynn, and C. F. Driscoll, Relaxation of 2D. Turbulence to Vortex Crystals, Phys. Rev. Lett. 75, 3277 (1995).
[11] D. A. Schecter, D. H. E. Dubin, K. S. Fine, and C. F. Driscoll, Vortex crystals from 2D Euler flow: Experiment and simulation, Phys. Fluids 11, 905 (1999).

[12] R. Kawahara and H. Nakanishi, Quasi-stationary states of twodimensional electron plasma trapped in magnetic field, J. Phys. Soc. Jpn. 75, 054001 (2006).

[13] D. Lynden-Bell, Statistical mechanics of violent relaxation in stellar systems, Mon. Not. R. Astron. Soc. 136, 101 (1967).

[14] Y. Y. Yamaguchi, One-dimensional self-gravitating sheet model and Lynden-Bell statistics, Phys. Rev. E 78, 041114 (2008).

[15] M. Joyce and T. Worrakitpoonpon, Quasistationary states in the self-gravitating sheet model, Phys. Rev. E 84, 011139 (2011).

[16] T. N. Teles, Y. Levin, R. Pakter, and F. B. Rizzato, Statistical mechanics of unbound two-dimensional self-gravitating systems, J. Stat. Mech. (2010) P05007.

[17] Y. Levin, R. Pakter, and F. B. Rizzato, Collisionless relaxation in gravitational systems: From violent relaxation to gravothermal collapse, Phys. Rev. E 78, 021130 (2008).

[18] F. P. C. Benetti, T. N. Teles, R. Pakter, and Y. Levin, Ergodicity Breaking and Parametric Resonances in Systems with Long-Range Interactions, Phys. Rev. Lett. 108, 140601 (2012).

[19] R. L. Gluckstern, Analytic Model for Halo Formation in High Current Ion Linacs, Phys. Rev. Lett. 73, 1247 (1994). 
[20] R. Pakter and Y. Levin, Core-Halo Distribution in the Hamiltonian Mean-Field Model, Phys. Rev. Lett. 106, 200603 (2011).

[21] Y. Levin, R. Pakter, F. B. Rizzato, T. N. Teles, and F. P. C. Benetti, Nonequilibrium statistical mechanics of systems with long-range interactions, Phys. Rep. 535, 1 (2014).

[22] X. Leoncini, T. L. Van Den Berg, and D. Fanelli, Out-ofequilibrium solutions in the XY-Hamiltonian mean-field model, Europhys. Lett. 86, 20002 (2009).

[23] P. de Buyl, D. Mukamel, and S. Ruffo, Self-consistent inhomogeneous steady states in Hamiltonian Mean-Field dynamics, Phys. Rev. E 84, 061151 (2011).

[24] A. C. Ribeiro-Teixeira, F. P. C. Benetti, R. Pakter, and Y. Levin, Ergodicity breaking and quasistationary states in systems with long-range interactions, Phys. Rev. E 89, 022130 (2014).

[25] F. P. C. Benetti, A. C. Ribeiro-Teixeira, R. Pakter, and Y. Levin, Nonequilibrium Stationary States of 3D Self-Gravitating Systems, Phys. Rev. Lett. 113, 100602 (2014).

[26] S. Ogawa and Y. Y. Yamaguchi, Nonlinear response for external field and perturbation in the Vlasov system, Phys. Rev. E 89, 052114 (2014).

[27] C. Lancellotti and J. J. Dorning, Critical Initial States in Collisionless Plasmas, Phys. Rev. Lett. 81, 5137 (1998).

[28] C. Lancellotti and J. J. Dorning, Time-asymptotic wave propagation in collisionless plasmas, Phys. Rev. E 68, 026406 (2003).

[29] C. Lancellotti and J. J. Dorning, Nonlinear Landau damping, Transp. Theory Stat. Phys. 38, 1 (2009).

[30] I. Y. Dodin and N. J. Fisch, Nonlinear Dispersion of Stationary Waves in Collisionless Plasmas, Phys. Rev. Lett. 107, 035005 (2011).

[31] I. Y. Dodin, On variational methods in the physics of plasma waves, Fusion Sci. Technol. 65, 54 (2014).

[32] L. Landau, On the vibrations of the electronic plasma, J. Phys. USSR 10, 25 (1946).
[33] S. Ogawa, A. Patelli and Y. Y. Yamaguchi, Non-mean-field critical exponent in a mean-field model: Dynamics versus statistical mechanics, Phys. Rev. E 89, 032131 (2014).

[34] S. Ogawa and Y. Y. Yamaguchi, Landau-like theory for universality of critical exponents in quasistationary states of isolated mean-field systems, Phys. Rev. E 91, 062108 (2015).

[35] S. Inagaki and T. Konishi, Dynamical stability of a simple model similar to self-gravitating systems, Publ. Astron. Soc. Jpn. 45, 733 (1993).

[36] M. Antoni and S. Ruffo, Clustering and relaxation in Hamiltonian long-range dynamics, Phys. Rev. E 52, 2361 (1995).

[37] J. Barré and Y. Y. Yamaguchi, Small traveling clusters in attractive and repulsive Hamiltonian mean-field models, Phys. Rev. E 79, 036208 (2009).

[38] T. O' Neil, Collisionless Damping of Nonlinear Plasma Oscillations, Phys. Fluids 8, 2255 (1965).

[39] J. H. Jeans, On the theory of star-streaming and the structure of the universe, Mon. Not. R. Astron. Soc. 257, 70 (1915).

[40] P. de Buyl, Numerical resolution of the Vlasov equation for the Hamiltonian Mean-Field model, Commun. Nonlinear Sci. Numer. Simulat. 15, 2133 (2010).

[41] A. Patelli, S. Gupta, C. Nardini, and S. Ruffo, Linear response theory for long-range interacting systems in quasistationary states, Phys. Rev. E 85, 021133 (2012).

[42] S. Ogawa and Y. Y. Yamaguchi, Linear response theory in the Vlasov equation for homogeneous and for inhomogeneous quasistationary states, Phys. Rev. E 85, 061115 (2012).

[43] P. de Buyl, G. De Niinno, D. Faneli, C. Nardini, A. Patelli, F. Piazza, and Y. Y. Yamaguchi, Absence of thermalization for systems with long-range interactions coupled to a thermal bath, Phys. Rev. E 87, 042110 (2013).

[44] J. Barré, A. Olivetti, and Y. Y. Yamaguchi, Dynamics of perturbations around inhomogeneous backgrounds in the HMF model, J. Stat. Mech. (2010) P08002. 